\title{
Abelson family kinases regulate Frizzled planar cell polarity signaling via Dsh phosphorylation
}

\author{
Jaskirat Singh, ${ }^{1}$ Wang A. Yanfeng, ${ }^{1}$ Luca Grumolato, ${ }^{2}$ Stuart A. Aaronson, ${ }^{2}$ and Marek Mlodzik ${ }^{1,3}$ \\ ${ }^{1}$ Department of Developmental and Regenerative Biology, Mount Sinai School of Medicine, New York, New York 10029, \\ USA; ${ }^{2}$ Department of Oncological Sciences, Mount Sinai School of Medicine, New York, New York 10029, USA
}

\begin{abstract}
Abelson (Abl) family tyrosine kinases have been implicated in cell morphogenesis, adhesion, motility, and oncogenesis. Using a candidate approach for genes involved in planar cell polarity (PCP) signaling, we identified Drosophila $\mathrm{Abl}$ (dAbl) as a modulator of Frizzled(Fz)/PCP signaling. We demonstrate that $d A b l$ positively regulates the Fz/Dishevelled (Dsh) PCP pathway without affecting canonical Wnt/Wg-Fz signaling. Genetic dissection suggests that Abl functions via Fz/Dsh signaling in photoreceptor R3 specification, a well-established Fz-PCP signaling readout. Molecular analysis shows that dAbl binds and phosphorylates Dsh on Tyr473 within the DEP domain. This phosphorylation event on Dsh is functionally critical, as the equivalent DshY473F mutant is nonfunctional in PCP signaling and stable membrane association, although it rescues canonical Wnt signaling. Strikingly, mouse embryonic fibroblasts (MEFs) deficient for Abl1 and Abl2/Arg genes also show reduced Dvl2 phosphorylation as compared with control MEFs, and this correlates with a change in subcellular localization of endogenous Dvl2. As in Drosophila, such Abl-deficient MEFs show no change in canonical Wnt signaling. Taken together, our results argue for a conserved role of $\mathrm{Abl}$ family members in the positive regulation of Dsh activity toward Fz-Dsh/PCP signaling by Dsh phosphorylation.
\end{abstract}

[Keywords: Drosophila; Abl; Dsh; Dvl; Fz; cell polarity]

Supplemental material is available at http://www.genesdev.org.

Received June 18, 2010; revised version accepted August 16, 2010.

Epithelia are generally polarized in the apical-basal axis, and can also be polarized within the plane of an epithelium. This latter polarization is referred to as tissue polarity or planar cell polarity (PCP). In Drosophila, PCP is manifest in almost all adult tissues, including the eye, wing, thorax, and abdomen (Klein and Mlodzik 2005; Lawrence et al. 2007; Seifert and Mlodzik 2007). In vertebrates, PCP signaling is, for example, associated with convergent extension, neurulation, skin-hair patterning, or the orientation of sensory hair cells of the cochlea (inner ear) (Wang and Nathans 2007). Moreover, PCP has been linked to several diseases, including neural tube closure defects, polycystic kidney disease (PKD), and ciliopathies in general (Simons and Mlodzik 2008). PCP has been studied mostly in Drosophila, and is controlled by two sets of conserved core PCP factors: (1) the Frizzled (Fz)/ PCP group, which includes Fz, Strabismus (stbm/Vang), Flamingo (Fmi), Dishevelled (Dsh), Prickle (Pk), and Diego (Dgo); and (2) the Fat/Dachsous (Ds) group containing Fat, Ds, Four-jointed, Dachs, and Approximated (Lawrence

${ }^{3}$ Corresponding author.

E-MAIL Marek.Mlodzik@mssm.edu; FAX (212) 860-1174.

Article published online ahead of print. Article and publication date are online at http://www.genesdev.org/cgi/doi/10.1101/gad.1961010. et al. 2007; Seifert and Mlodzik 2007; Wu and Mlodzik 2009). All of these factors are conserved in vertebrates, suggesting common mechanisms by which PCP is established across all metazoa (Wang and Nathans 2007).

Some members of the Fz/PCP group, most prominently $\mathrm{Fz}$ and Dsh, are also essential components of canonical Wnt-Fz signaling, regulating gene expression via $\beta$-catenin stabilization (MacDonald et al. 2009). The multidomain protein Dsh (Dvl1, Dvl2, and Dvl3 in mammals) is an important component of $\mathrm{Fz} / \mathrm{PCP}$ signaling as well as of the $\mathrm{Fz} / \beta$-catenin pathway, but the regulation of canonical and PCP Wnt signaling at the level of Dsh still remains poorly understood. In canonical Wnt signaling, Dsh phosphorylation inhibits the APC-Axin-GSK3 complex, leading to $\beta$-catenin stabilization. In contrast, in PCP signaling, Dsh is stably recruited to the membrane, where it can lead to the activation of Rho and Rac family GTPases as downstream effectors. Dsh contains three highly conserved domains: the DIX, PDZ, and DEP domains. The DIX domain of Dsh functions exclusively in canonical signaling, while the PDZ domain is required in both pathways and is important for protein-protein interaction. The DEP domain is required mainly in PCP signaling and is thought to mediate activation of the small Rho family GTPases and downstream effectors (Boutros and Mlodzik 1999; 
Wallingford and Habas 2005). The DEP domain also plays a central role in the cytoplasmic-to-membrane translocation of Dsh in response to $\mathrm{Fz} / \mathrm{PCP}$ signaling.

In a search for novel regulatory factors involved in modulating the activity of core PCP factors and associated signaling, we used a candidate gene approach, analyzing mutants that have rough eye phenotypes comparable with those associated with core PCP mutants. One candidate identified was the Drosophila Abelson nonreceptor tyrosine kinase gene (dAbl) (Henkemeyer et al. 1987). Loss of $d A b l$ leads to developmental defects, which include pupal lethality, rough eyes, reduced fertility, and reduced life span (Bennett and Hoffmann 1992). In Drosophila embryos, Abl regulates adherens junction stability, and actin organization during apical constriction via the actin regulator Enabled (Ena) (Grevengoed et al. 2001; Fox and Peifer 2007). In the eye, dAbl is expressed in all developing photoreceptors, and has been shown recently to be important for photoreceptor axon targeting in the brain (Bennett and Hoffmann 1992; Xiong et al. 2009).

Here we demonstrate that $d A b l$ loss of function (LOF) displays classical PCP phenotypes. We show that it positively modulates the Fz/Dsh PCP pathway without affecting canonical Wnt/Wg-Fz signaling. Genetically, dAbl acts in the R3 photoreceptor in a Dsh-dependent manner. We also show that dAbl binds and phosphorylates Dsh. This phosphorylation maps to the DEP domain of Dsh, a key domain for PCP signaling, on Tyr473. The phosphorylation event is functionally critical for Dsh PCP function, as the equivalent DshY473F mutant is nonfunctional in PCP signaling and also does not show the PCP-specific asymmetric membrane association, although it still rescues canonical Wnt signaling. Furthermore, mouse embryonic fibroblasts (MEFs) deficient for Abl1 and Abl2/Arg also show reduced Dvl2 and Dvl3 phosphorylation, which correlates with a change in the subcellular localization of Dvl2. Of note, such Abl-deficient MEFs show no change in canonical Wnt signaling. In summary, our results highlight an unexplored role of Abl family kinases in positively modulating Dsh activity toward the Fz-Dsh/PCP pathway by affecting Dsh phosphorylation.

\section{Results}

\section{$\mathrm{dAbl} L O F$ alleles and gain of function display PCP defects}

To study the role of dAbl in cell fate specification during eye development, we analyzed several $d A b l$ alleles in trans to a deficiency removing $d A b l$. In wild-type eyes, the Fz/PCP pathway differentially specifies photoreceptors R3 and R4 in developing ommatidia. This step is followed by a $90^{\circ}$ rotation of each ommatidial cluster toward the dorsal-ventral midline, or equator (Seifert and Mlodzik 2007). This results in a characteristic arrangement of ommatidia, where the dorsal half is the mirror image of the ventral half (Fig. 1A). In eyes with compromised $d A b l$ function, we observed a significant portion (ranging between 53\% and 66\%) of ommatidia with photoreceptor loss (as noted previously; Henkemeyer et al. 1987) and also ommatidia that displayed classical PCP
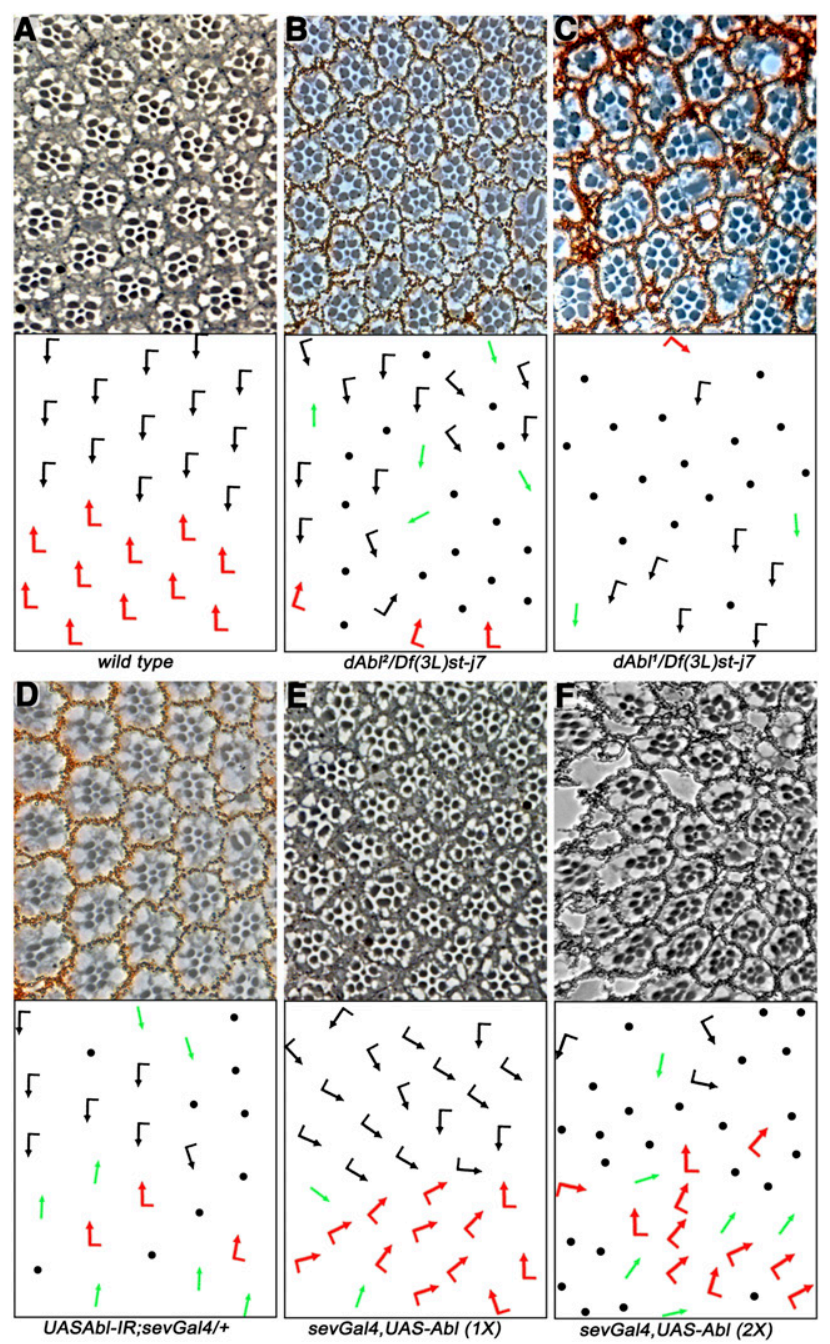

Figure 1. $d A b 1 \mathrm{LOF}$ and gain of function gives PCP phenotypes. All panels show tangential adult eye sections around the equator with anterior left and dorsal up. The bottom panels show schematic representations reflecting the polarity and orientation of individual ommatidia: Black arrows represent the dorsal ommatidia, red arrows represent ventral ommatidia, green arrows represent symmetrical clusters (that have lost chirality and the distinction between the R3/R4 cells), and black dots represent ommatidia that have a reduced number of photoreceptors and cannot be scored for PCP. (A) Wild-type eye; note the regular arrangement and mirror-image alignment of the dorsal and ventral halves around the equator. $(B) d A b I^{2}$ / $D f(3 L) s t-j 7$ eye; note the PCP defects along with loss of photoreceptors. (C) $d A b 1^{1} / D f(3 L) s t-j 7$ eye; again displaying PCP defects along with strong loss of photoreceptors, suggesting a requirement of $\mathrm{dAbl}$ in the survival of photoreceptors; the more severe loss of $\mathrm{R}$ cells in the $d A b l^{1}$ allele makes it difficult to score for PCP defects. (D) Expression of UAS-dAbl-IR under sevGal4 control (sevGal4, UAS-dAbl-IR in the presence of $U A S-d c r 2)$ at $18^{\circ} \mathrm{C}$ yields PCP defects with a reduced loss of photoreceptors as compared with $d A b 1^{1} .(E, F)$ Overexpression of dAbl using sevGal4 (sevGal4, UAS-dAbl) causes strong rotation phenotypes and PCP defects. $(F)$ This effect is dosedependent and is stronger with two copies $(2 \times$ sevGA14, UAS$d A b 1)$. See also Supplemental Table S1. 
defects (with PCP defects in $15 \%-20 \%$ of scorable ommatidia) (Fig. 1B,C). Generally, the stronger alleles showed a greater loss of photoreceptors, rendering many ommatidia unscorable, compared with weaker alleles where a larger fraction of ommatidia could be scored for PCP defects $\left(d A b l^{2}\right.$ is a weaker allele than $d A b 1^{1}$, a strong hypomorph) (Fig. 1C; see also Supplemental Table S1). These data suggested that $d A b l$ plays a role not only in photoreceptor cell survival, but also in the PCP-associated process of R3/ R4 cell fate specification. In order to bypass the cell survival defects associated with $d A b l \mathrm{LOF}$, we expressed dAbl-RNAi (dAbl-IR) under sevenlessGal4 (sevGal4), specifically driving expression in a subset of developing photoreceptors and, in particular, at high levels in R3 and R4 during PCP establishment. Strikingly, sevGal4, dAbl-IR resulted in many ommatidia displaying chirality defects and symmetrical clusters $(53.3 \% \pm 9.1 \%$ of scorable ommatidia) (Fig. 1D), confirming that $d A b l$ is required for PCP establishment in the eye. Furthermore, expression of $d A b l-I R$ using engrailedGal4 (enGal4, expressed in the posterior compartment of the wing) resulted in growthrelated defects (data not shown) along with wing PCP/ cellular hair orientation defects (Supplemental Fig. S1B,B'), suggesting a conserved role of dAbl in PCP signaling.

As $d A b l$ LOF resulted in eye PCP phenotypes, we next tested whether overexpression of dAbl has an effect on R3/R4 cell fate specification. Expression of dAbl (UAS$d A b l)$ under sevGal4 control caused mild PCP defects (Fig. 1E; Supplemental Table S1), with many ommatidia showing misrotations. To test if this effect is dosage-sensitive, we expressed dAbl using two copies each of UAS-dAbl and sevGal4. Such increased expression resulted in strong PCP phenotypes with many symmetrical clusters, along with frequent loss of photoreceptors (Fig. 1F; Supplemental Table S1).

\section{$\mathrm{dAbl}$ is required in $R 3$ and $R 4$ cell fate specification}

To identify the requirement of $d A b l$ in specific photoreceptors/R cells, we analyzed phenotypically wild type, yet genetically mosaic, ommatidia for their composition. In wild-type eye clones (marked by $W^{-}$) the ratio between ommatidia with $w^{-}$and $w^{+} \mathrm{R}$ cells for a specific outer photoreceptor is 0.5 for each photoreceptor subtype, because wild-type ommatidia photoreceptors can be either $W^{-}$or $w^{+}$with equal probability. In contrast, $d A b l$ clones displayed a reduced ratio for mutant R3 $(r=0.27)$ and $\mathrm{R} 4(r=0.25)$ cells (Fig. $2 \mathrm{~A}, \mathrm{~B})$, implying that $d A b l$ is specifically required in $\mathrm{R} 3 / \mathrm{R} 4$ photoreceptors for their fate induction, specification, and/or survival. To further define the $d A b l$ requirement in PCP establishment, we performed a mosaic analysis of scorable ommatidia (mosaic clusters with normal R-cell complement) that display chirality defects (symmetrical clusters or chirality flips). Such clusters comprised $18.4 \%$ of scorable ommatidia (examples are shown in Fig. 2A, see green and red arrows in the schematic). In these mosaics (displaying a PCP defect), 94\% were mutant for $d A b 1$ in R3 and/or $\mathrm{R} 4$, whereas the genotype $\left(d A b 1^{-}\right.$vs. $\left.d A b I^{+}\right)$of the other $\mathrm{R}$ cells did not contribute to the PCP phenotype (data not shown). This behavior of dAbl mosaic clusters is very similar to core PCP genes (Klein and Mlodzik 2005; Seifert and Mlodzik 2007), indicating that $d A b l$ is indeed required in R3 and R4 for PCP establishment.

To further confirm the role of $d A b 1$ in R3/R4 fate specification, we next analyzed $d A b l^{4}$ mutant clones in third instar eye imaginal discs with the molecular R4 marker $m \delta>0.5$-LacZ (in third instar eye discs, $m \delta 0.5$ $L a c Z[m \delta>L a c Z]$ is first expressed in both R3/R4 cells at low levels and then up-regulated specifically in R4) (Fig. 2C-C"; Cooper and Bray 1999). Strikingly, $m \delta>L a c Z$ expression was reduced or lost in $d A b l$ mutant ommatidia
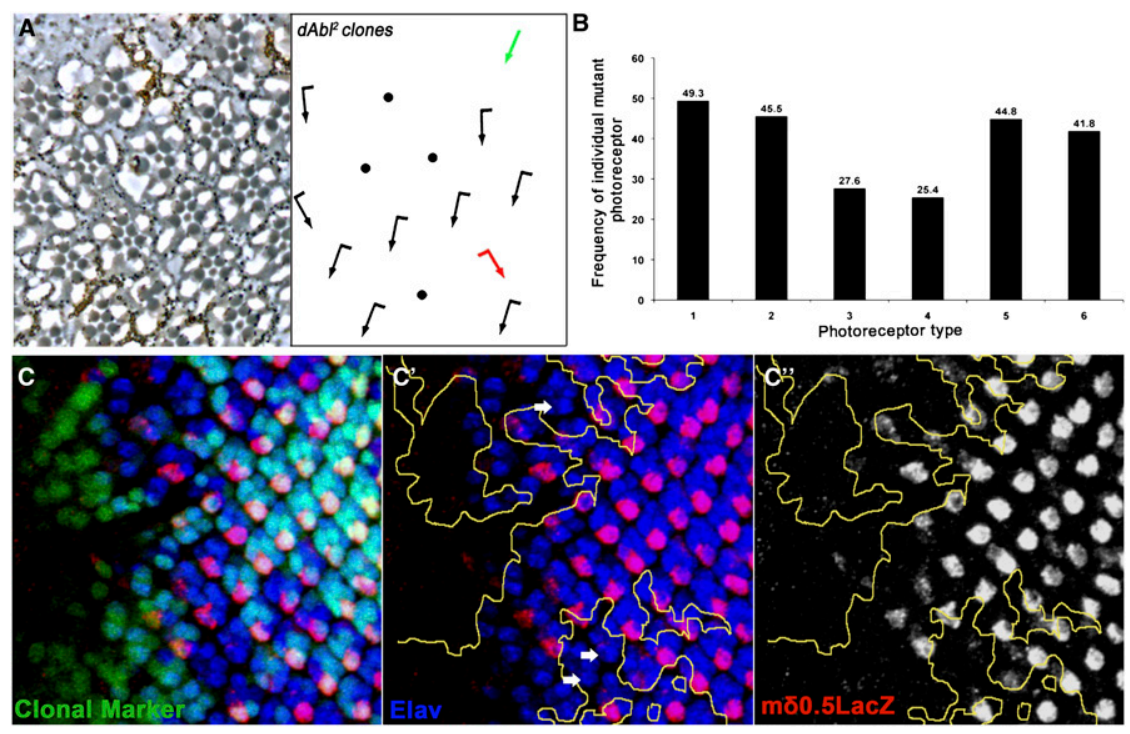

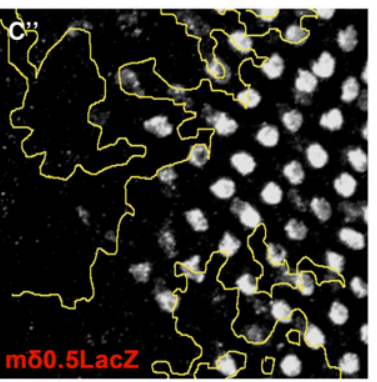

Figure 2. $d A b l$ is required in $\mathrm{R} 3$ and $\mathrm{R} 4$ for their fate specification. (A) $d A b l^{2}$ mosaic eye (mutant tissue marked by absence of pigment). Note ommatidia that, when mutant for $d A b 1$ in R3 and/or R4, show PCP defects. $(B)$ Quantification of mosaic ommatidia, which are phenotypically wildtype but mutant for any of the R1-R6 outer photoreceptors (random frequency reflecting no requirement is $0.5 ; n=152$, nine eyes analyzed). If $d A b 1$ is required for the specification of a specific photoreceptor type, the number of phenotypically normal ommatidia mutant for that photoreceptor will be significantly lower than the number of ommatidia wild-type for that photoreceptor, leading to a decreased ratio. $d A b l$ mutant R3 and R4 cells have a significantly reduced frequency in ommatidia with wildtype appearance, suggesting a requirement of $d A b 1$ in the R3/R4 cells and PCP signaling. $\left(C-C^{\prime \prime}\right)$ Confocal projections of $d A b I^{4}$ clones in third instar eye discs (mutant tissue marked by absence of GFP and outlined by yellow lines in $C^{\prime}, C^{\prime \prime}$ ). R4 cells are molecularly

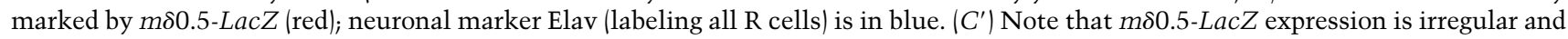
reduced/lost in the $d A b l$ mutant tissue (white arrows), suggesting a failure in normal R3/R4 specification. 
(21.2\% of mutant clusters) (Fig. 2C-C"), suggesting a failure in proper R4-cell subtype specification (mutant $\mathrm{R}$ cells still expressed the common R-cell marker Elav). Similar suppression of $m \delta>L a c Z$ was also observed in $d A b l^{2}$ clones (Supplemental Fig. S2A-A"). In summary, we conclude that loss of dAbl results in PCP defects in the eye with a genetic requirement in the $\mathrm{R} 3 / \mathrm{R} 4$ photoreceptors.

$\mathrm{fz}$ and dsh phenotypes are modified by dAbl

To gain insight into how dAbl mediates PCP establishment, we performed genetic interactions with core PCP components. Direct overexpression of Fz or Dsh from the $s e v$ enhancer/promoter sev-Fz and sev-Dsh, respectively, resulted in strong PCP phenotypes characterized by a high frequency of R3-R3 symmetrical clusters (Fig. 3A,C; Supplemental Table S2; e.g., see also Boutros et al. 1998). Strikingly, removing a genomic copy of $d A b l$ (using either $d A b I^{2}$ or $d A b I^{4}$ alleles) resulted in a dominant suppression of both the $s e v-F z$ and $s e v-D s h$ phenotypes (Fig. 3B,D; Supplemental Table S2). These data suggested that dAbl may function downstream from (or in parallel to) Fz/Dsh-PCP signaling, and that dAbl acts positively in regulating $\mathrm{Fz} /$ Dsh-PCP signaling activity. Of note, removing a genomic copy of $d A b l$ did not suppress sev-Fmi or sev-Stbm PCP phenotypes (Supplemental Fig. S3A-D; Supplemental Table S2), suggesting that its interaction is specific to $\mathrm{Fz}$ and Dsh and is not due to a general modification of core PCP members. We next tested an interaction of $d A b l$ with downstream components of Fz/Dsh-PCP signaling. The Drosophila rac genes have been implicated as downstream effectors of Dsh in PCP establishment (Fanto et al. 2000; Munoz-Descalzo et al. 2007). Overexpression of Racl using sevGal4 (sev > Rac) resulted in ommatidial rotation defects (Fig. 3E; Supplemental Table S2). Removing a copy of $d A b l$ did not modify sev $>$ Rac (Fig. 3F; Supplemental Table S2), suggesting that $d A b 1$ acts upstream of the rac genes. Taken together, these data suggest that dAbl acts at the level of or downstream from $\mathrm{Fz}$ and Dsh, but upstream of Dsh effectors, in PCP establishment.

To gain further insight into the role of $\mathrm{dAbl}$ in the Fz/DshPCP pathway, we performed mosaic analyses of $d A b l$ in a heterozygous $d s h^{1}$ background $\left(d s h^{1}\right.$ is a strong PCP-specific
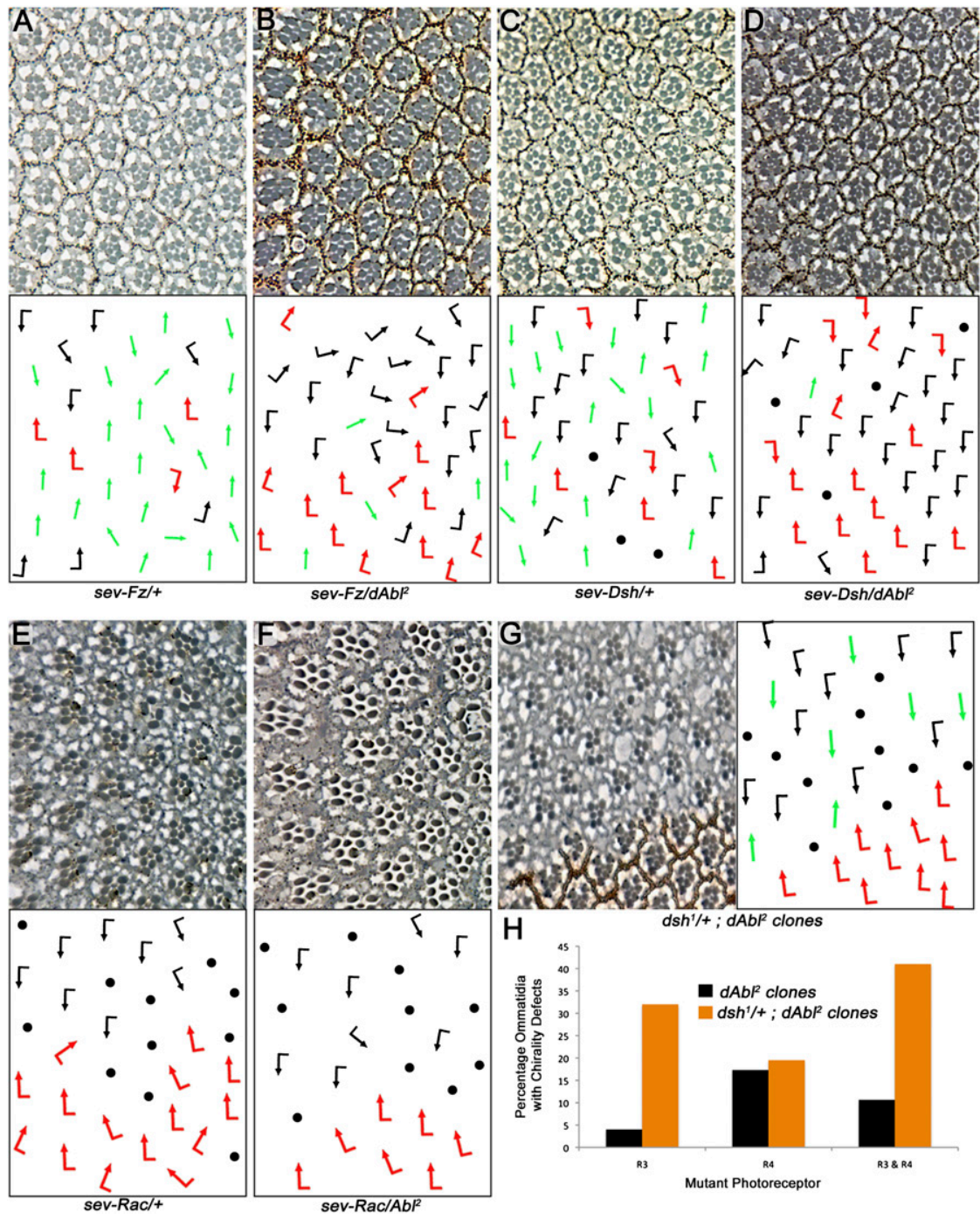

Figure 3. $d A b l$ genetically interacts with $\mathrm{Fz}$ and Dsh PCP phenotypes. All panels show tangential adult eye sections in areas around the equator (top panels) with respective schematic representations (bottom panels), except for $H$, which is a quantification. Arrows are as in Figure 1. (A) sev-Fz/+ eye, displaying a high frequency of symmetrical R3/R3-type ommatidia. (B) sev-Fz/+, $d A b 1^{2} /+$; note reduction in symmetrical ommatidia, indicating a strong genetic suppression of the $\mathrm{sev}-\mathrm{Fz}$ phenotype. (C) sev-Dsh/+ shows strong PCP defects with many symmetrical ommatidia. (D) sev-Dsh/+, $d A b 1^{2} /+$; note suppression of the number of symmetrical ommatidia; it is worth mentioning that the loss of R-cell features also seen in sev-Dsh (shown in C), which is mediated by canonical $\mathrm{Wg}$ signaling (Boutros et al. 1998), is not suppressed, suggesting a specific genetic suppression of the Dsh PCP function. (E) sev-Rac/+ ommatidia mostly show rotation defects along with loss of photoreceptors. (F) sev-Rac/+, $d A b l^{2} /+$ shows a very similar phenotype as $\mathrm{sev}$ $R a c /+$ alone, suggesting that $d A b l$ probably acts upstream of the Rac GTPAses. $(G)$ $d A b 1^{2}$ eye clones in a $d s h^{1} /+$ background $\left(d s h^{1}\right.$ is a PCP-specific allele). Strikingly, removing a genomic copy of $d s h$ enhances the $d A b 1^{2}$ PCP phenotype specifically in R3 photoreceptors, suggesting a function in R3 specification in a Dsh-dependent manner. $(H)$ Quantification of mosaic ommatidia in the $d A b l^{2}$ clones in the $d s h^{1} /+$ background (with focus on R3 and R4 genotypes). Note the increased requirement in R3 $\left(d A b l^{2}\right.$ clones in the $d s h^{1} /+$ background; orange bar) as compared with $d A b 1^{2}$ clones (black bars). 
allele not affecting the canonical Wnt/Wg pathway) (e.g., Boutros et al. 1998). Removing a genomic copy of $d s h$ $\left(d s h^{1} /+\right.$, which is phenotypically wild type by itself) in a $d A b l$ mutant clone background significantly enhanced PCP defects specifically when R3 was mutant for $d A b l$ (Fig. 3H, cf. black and orange bars when R3 is mutant), suggesting that dAbl has a R3-specific function that is Dsh-dependent (dsh is required in R3 for its PCP function) (Boutros et al. 1998). In contrast, when $d A b l$ function was compromised in R4 photoreceptors in a $d s h^{1} /+$ background, there was no change in the phenotype and requirement as compared with dAbl mutant clones (Fig. $3 \mathrm{H}$, cf. black and orange bars when only R4 is mutant). These data indicate that dAbl function in $\mathrm{R} 3$ is dependent on $\mathrm{Fz} /$ Dsh-PCP signaling and, in particular, on Dsh.

\section{dAbl modulates Fz/Dsh-PCP signaling but not Fz2-mediated canonical Wg signaling}

Our data suggest that dAbl can affect PCP establishment via Fz/Dsh signaling in $\mathrm{R} 3$ cells. Next we tested whether dAbl acts specifically in the Fz/Dsh-PCP pathway, or whether it also has an effect on canonical Wnt/Wg signaling. In Drosophila wings, overexpression of Fz or Fz2 with decapentaplegic-Gal4 (dpp-Gal4; driving expression in a stripe along the anterior-posterior/A-P compartment boundary) (see Fig. 4A) can be used to study the effects of gene function on canonical $\mathrm{Wg}$ and PCP signaling, respectively (e.g., Klein et al. 2006). Using this dppGal4 assay, we tested whether coexpression of dAbl with either Fz or Fz2 could modify their signaling effects. dppGal4-driven UAS- $d A b l$ alone (dppGal4 > dAbl) did not cause detectable phenotypes at $18^{\circ} \mathrm{C}$, and only very mild PCP defects at $29^{\circ} \mathrm{C}$ (Fig. 4B,C, respectively). Overexpression of $\mathrm{Fz}$ (dppGal4 > Fz) resulted in reorientation of wing hairs away from the $d p p$ expression domain; this effect was very mild at $18^{\circ} \mathrm{C}$ (Fig. 4D) and was strong at $25^{\circ} \mathrm{C}$ (Supplemental Fig. S4A; see also Jenny et al. 2005; Wu et al. 2008). Coexpression of dAbl and $\mathrm{Fz}(d p p G a 14>F z, d A b l)$ at $18^{\circ} \mathrm{C}$ resulted in a strong enhancement of the $d p p>F z$ effect (Fig. 4E), indicating a positive synergistic relationship between Fz-mediated PCP signaling and dAbl function. Accordingly, expression of $d A b l-I R$ strongly suppressed the $d p p>F z$ wing hair orientation phenotype (Supplemental Fig. S4, cf. direction of arrows in A and B). In contrast, dAbl did not affect the $d p p>$ Fz2-mediated canonical $\mathrm{Wg}$ signaling phenotype (Fig. 4F, G, note no change in the number of extra margin bristles, as induced by increased $\mathrm{Wg} / \mathrm{Fz} 2$ signaling in the presence of dAbl; see Supplemental Fig. S5 for quantification). Accordingly, mutant clones of $d A b l^{2}$ in the wing did not affect wing margin development, as seen by normal expression of the Wg signaling target Senseless in mutant cells (Fig. 4H-H"). This is consistent with phenotypic analyses of embryos completely lacking both maternal and zygotic $d A b l$, which show no canonical Wg signaling-associated defects (Grevengoed et al. 2001). Taken together, these data indicate that dAbl functions specifically to promote $\mathrm{Fz} / \mathrm{Dsh}-\mathrm{PCP}$ signaling and does not affect canonical Wg signaling.

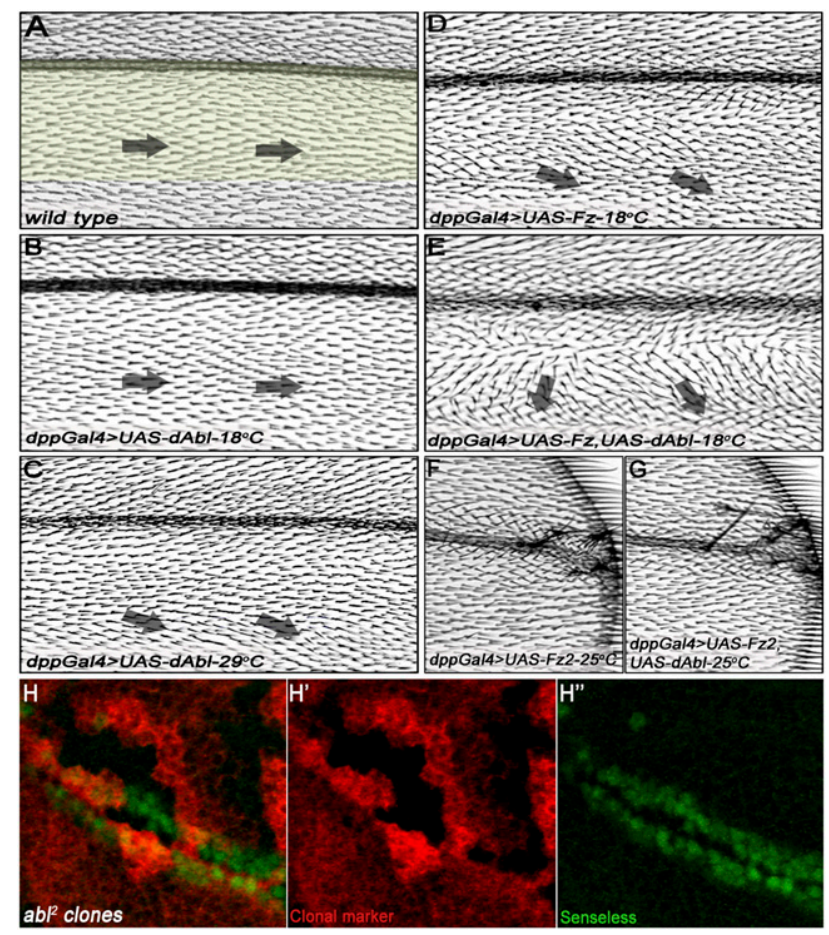

Figure 4. dAbl enhances Fz-mediated gain-of-function PCP phenotypes, but not Fz2-mediated canonical Wg signaling effects in the wing. All panels show adult wings oriented with proximal toward left and anterior up. (A) Wild-type wing highlighting the $d p p$ expression domain; note the orientation of cellular hairs from proximal to distal. $(B)$ dppGal4 > UAS$d A b 1$ wing $\left(18^{\circ} \mathrm{C}\right)$ does not show any effects. $(C)$ dpp Gal4 > UAS$d A b l$ wing $\left(29^{\circ} \mathrm{C}\right)$ displays mild PCP defects, with cellular hairs pointing slightly away from the $d p p$ expression domain. $(D)$ dppGal4 > UAS-Fz wing $\left(18^{\circ} \mathrm{C}\right)$ shows very mild PCP phenotypes with cellular hairs pointing slightly away from the $d p p$ domain (posterior to the expression domain), indicated by black arrows. (E) dppGal4 >UAS-Fz, UAS-dAbl wing $\left(18^{\circ} \mathrm{C}\right)$ shows a synergistic enhancement of the PCP phenotypes of $\mathrm{Fz}$ and $\mathrm{dAbl}$; note the markedly increased reorientation of wing hairs flanking the $d p p$ expression domain (examples highlighted by black arrows). This effect suggests a positive relationship between dAbl and $\mathrm{Fz}$, both promoting $\mathrm{Fz} / \mathrm{PCP}$ signaling. $(F)$ dppGal4 > UAS-Fz2 wing $\left(25^{\circ} \mathrm{C}\right)$ showing ectopic margin bristles within the $d p p$ domain close to the source of $\mathrm{Wg}$ (margin), reflecting a gain-of-function Wg signaling phenotype. (G) dppGal4 > UAS-Fz2, UAS- $d A b 1$ wing $\left(25^{\circ} \mathrm{C}\right)$; note that there is no change in the number of ectopic margin bristles as compared with $F$, suggesting that dAbl does not modulate canonical Wg signaling activity. $\left(H-H^{\prime \prime}\right)$ Confocal projections of $d A b l^{2}$ clones in third instar wing discs (mutant tissue marked by absence of LacZ; red in $H, H^{\prime}$ ) stained for the Wg signaling target gene senseless (anti-Sens; green in $H, H^{\prime \prime}$ ). Expression of senseless remains unchanged inside the $\mathrm{dAbl}^{2}$ clones.

\section{dAbl binds and phosphorylates Dsh}

As our genetic data suggested that dAbl modulates Fz/DshPCP signaling, we wished to establish a mechanistic link between dAbl and PCP pathway components. Abl family kinases are nonreceptor tyrosine kinases that can phosphorylate multiple substrates. Thus, we tested whether dAbl can physically bind and phosphorylate Dsh, which is 
a multidomain cytoplasmic protein with several conserved tyrosine residues (Boutros and Mlodzik 1999; Wallingford and Habas 2005). Although previous studies have identified a host of serine/threonine kinases that can phosphorylate Dsh to activate both the canonical and PCP pathways (Willert et al. 1997; Sun et al. 2001; Cong et al. 2004; Klein et al. 2006; Strutt et al. 2006), tyrosine phosphorylation of Dsh (or its mammalian homologs, Dvl1-3) has not yet been reported. To test for a potential physical interaction of dAbl and Dsh and/or phosphorylation of Dsh by dAbl, we cotransfected S2 cells with Dsh-Myc- and dAbl-HA-tagged constructs. Immunoprecipitation of Dsh-Myc led to a specific pull-down of dAbl-HA, suggesting a physical interaction between these proteins (Fig. 5A, cf. negative controls). Furthermore, we also detected coimmunoprecipitation of dAbl and Dsh when coexpressed in 293T cells (data not shown). We next investigated whether dAbl can phosphorylate Dsh. Immunoprecipitated Dsh-Myc was tyrosine phosphorylated when dAbl was cotransfected (Fig. 5B, cf. lanes 2 and 1), while coexpression of a kinase-dead mutant of dAbl (dAbl-KD) did not lead to phosphorylation of Dsh.

To confirm the binding, and to define the region of Dsh that interacts physically with dAbl, we performed glutathione S-transferase (GST) pull-down assays. Dsh consists of three conserved domains-DIX, PDZ, and DEP domainseach with specific requirements in either canonical or PCP signaling or both (Boutros and Mlodzik 1999; Wallingford and Habas 2005). Purified GST-Dsh fragments (Fig. 5C; Jenny et al. 2005) covering the distinct domains of Dsh were tested for their binding capacity to pull down in vitro translated dAbl. These experiments confirmed the cell culture data that dAbl can bind Dsh (Fig. 5D, lane 4 ), and the main binding mapped to the Extd.PDZ region comprising the proline-rich region C-terminal to the PDZ domain and a stretch of basic and $\mathrm{S} / \mathrm{T}$ residues $\mathrm{N}$-terminal to it (Fig. 5D [lane 7], C), while the PDZ domain alone did not show any binding (Fig. 5D, lane 6).

We next used in vitro kinase assays to determine the region of Dsh that is phosphorylated by dAbl. These experiments revealed that the DEP/C-term fragment (Fig. $5 \mathrm{E})$ was strongly phosphorylated by purified Abl kinase (Fig. 5E [lane 5, GST-Crk, a known Abl substrate, was used as a positive control], E [lane 6]). Of note, for the same level of expression of the Extd.PDZ and DEP/C-term, the level of phosphorylation was much higher with the DEP/C-term fragment (Fig. 5E, cf. lanes 4 and 5). Using purified PDZ and DEP domains of mouse Dvl1, the result was confirmed and further narrowed down to the DEP domain proper (Supplemental Fig. S6; data not shown). Significantly, the DEP domain of Dsh has been implicated in the activation of downstream $\mathrm{Fz} / \mathrm{PCP}$ signaling events and $\mathrm{PCP}$-specific membrane recruitment of Dsh (Boutros et al. 1998; Wu et al. 2008).

\section{Tyr473 is essential for Dsh function in PCP signaling} and is phosphorylated by $\mathrm{Abl}$

In the Dsh DEP/C-term region, there are three tyrosine residues within the DEP domain (Fig. 6A, Tyr473 [red] and Tyr474 [blue] are the two conserved tyrosines), and an
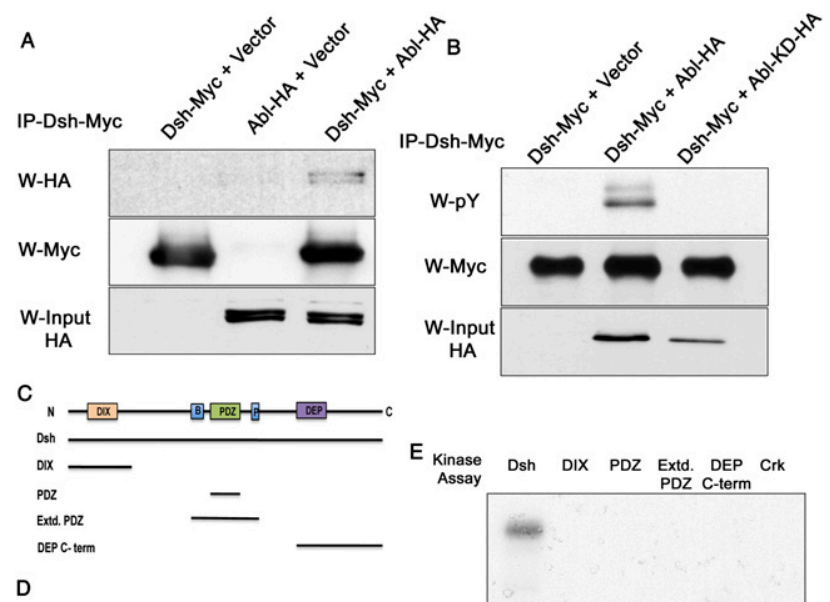

D
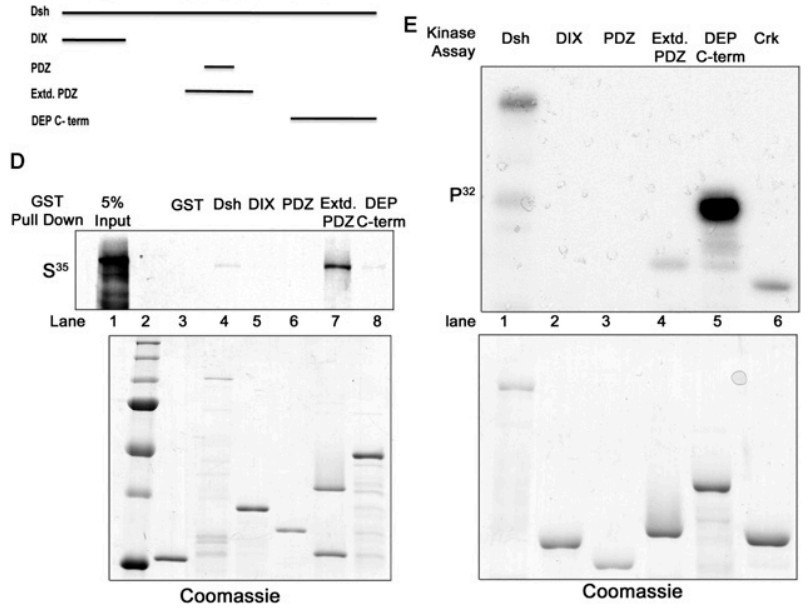

Figure 5. Abl binds Dsh and phosphorylates its C-terminal region. (A) Coimmunoprecipitation of dAbl-HA tag and DshMyc tag. S2 cells were cotransfected with either Dsh-Myc or dAbl-HA or both, and were immunoprecipitated with anti-Myc to pull down Dsh. Coexpression of dAbl and Dsh is accompanied by a coimmunoprecipitation of dAbl by Dsh, suggesting that they form a complex. (B) dAbl phosphorylates Dsh in 293T cells. Cells expressing Dsh-Myc along with dAbl-HA or dAblKD-HA (kinase-dead version of dAbl) were immunoprecipitated for Dsh and probed with phosphotyrosine antibody. (Top panel) Expression of dAbl along with Dsh results in phosphorylation of Dsh (shown in lane 2), while Dsh alone or Dsh with dAbl-KD does not show tyrosine phosphorylation. The middle panel shows the amount of Dsh protein pulled down in each lane, while the bottom panel shows the expression of dAbl and dAblKD. (C) Schematic representation of Dsh highlighting DIX, $\mathrm{PDZ}$, and DEP domains along with the basic region (B) and proline-rich region $(\mathrm{P})$. The various GST-tagged Dsh truncations (covering all conserved domains) are indicated below the schematic as black lines. $(D)$ GST pull-down assays using purified GST-Dsh truncations (see $C$ ) and in vitro translated dAbl kinase. The top panel shows an autoradiograph image with full-length Dsh and the Extd.PDZ fragment and DEP/C-term region of Dshbinding dAbl. Note that Dsh-Extd.PDZ shows a strong binding to dAbl, while full-length and DEP/C-term Dsh show a weaker binding. The other GST-Dsh fusion proteins did not bind, serving as negative controls. The bottom panel shows a Coomassie-stained gel to reveal loading amounts. (E) In vitro kinase assay with purified $\mathrm{Abl}$ and GST-Dsh truncations (as in $C, D$ ). The top panel shows an autoradiograph of a kinase assay, where full-length Dsh, Extd.PDZ, and DEP/C-term fragments show tyrosine phosphorylation. (Right lane) GST-Crk was included as a positive control. The bottom panel shows a Coomassie gel of the various GST fusions as loading control. 
additional five Tyr residues in the adjacent C-terminal sequences (Fig. 6D). We wished to determine whether any of these tyrosines are physiologically important for Dsh function in vivo. To this end, we generated Dsh transgenes using an endogenous $d s h$ expression cassette that was shown to rescue $d s h$-null mutant animals (Axelrod 2001). The equivalent $d s h-D s h G F P$-wt transgene fully rescued the $d s h^{v 26}$-null allele (Fig. 6B; data not shown). Similarly, most of the Tyr mutations tested fully rescued the $d s h^{\text {v26 }}$-null allele (e.g., $d s h-D s h G F P-Y 474 F$ or $d s h$ DshGFPY525,528F) (Fig. 6D; data not shown). In contrast, dsh-DshGFP-Y473F did not rescue the function of Dsh in PCP signaling (Fig. 6C,D; data not shown), although it maintained Dsh function for canonical Wnt signaling as it rescued the null allele to viability (data not shown). As such, DshY473F behaved very much like the PCP-specific $d s h$ allele $d s h^{1}$ (Boutros et al. 1998), highlighting the importance of this residue in PCP signaling.

As stable membrane association of Dsh (in a Fz-dependent manner) is a hallmark of PCP signaling (Axelrod
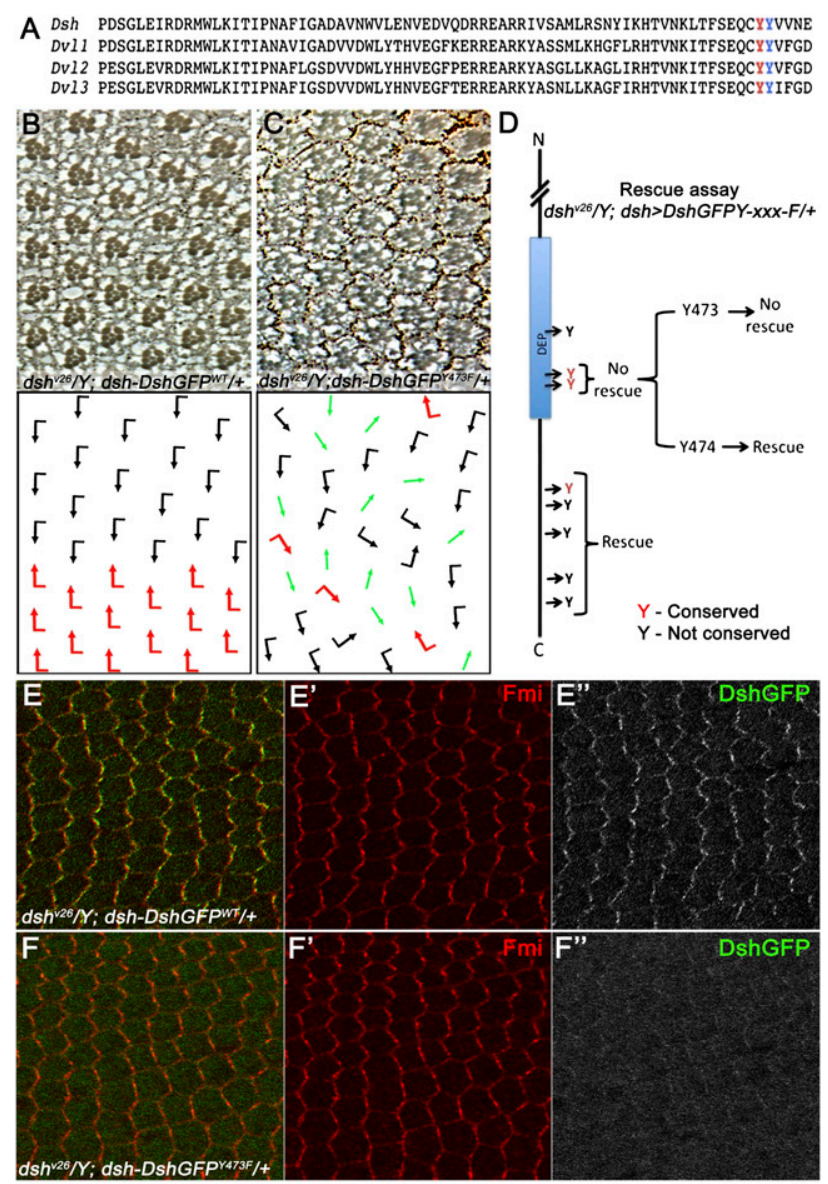

G

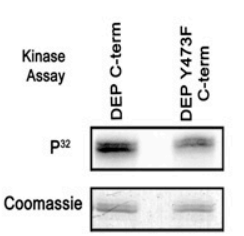

G'

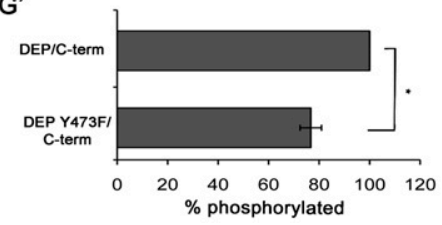

2001; Simons et al. 2009), we next tested whether Tyr473 can affect membrane localization of Dsh. Strikingly, DshY473F showed a markedly reduced or even absent membrane association during the critical stages of PCP signaling; e.g., during pupal wing PCP establishment (Fig. 6, cf. F-F" and wild-type Dsh in E-E", of note, DshY473F is as stable as Dsh ${ }^{\mathrm{WT}}$, as judged by quantification of fluorescence in DshY473F vs. Dsh ${ }^{\mathrm{WT}}$ pupal wing cells [using ImageJ software] or Western blot analyses; data not shown). This effect is again very similar to the $\mathrm{Dsh}^{1}$ mutant protein itself or other PCP-specific lesions in Dsh (Axelrod 2001; Simons et al. 2009).

Having established that the Tyr473 is physiologically important for Dsh function in PCP, we tested whether this site is a preferential Abl target in Dsh. We performed in vitro kinase assays by mutating Tyr473 within the DEP/ C-term protein fragment (established in Fig. 5E). Strikingly, DEP-Y473F/C-term showed a marked reduction of phosphorylation as compared with wt-DEP/C-term or the other point mutations (Fig. 6G, see also quantification in

Figure 6. Tyr473 in the DEP domain is essential for Dsh function in PCP signaling and is phosphorylated by Abl. $(A)$ Protein sequence alignment of Dsh DEP domain from Drosophila Dsh and three mammalian Dvls, showing two conserved tyrosine residues (Tyr473 and Tyr474) within the DEP domain. $(B-D)$ Rescue assay using the $d s h-D s h G F P$ expression cassette in a $d s h$-null background: $(B) d s h^{\mathrm{v} 26} / Y$; $d s h-D s h G F P^{W T} /+$ flies were fully rescued and their eyes displayed wild-type PCP features. Note that $d s h-D s h G F P^{W T}$ fully rescued all aspects of the $d s h^{\text {v26. }}$. (C) $d s h^{\mathrm{v} 26} / Y$; dsh-DshGFP $\mathrm{Y}^{\mathrm{4} 73 F} /+$ : Note that, while $d s h-$ $D s h G F P^{Y 473 F} /+$ rescued lethality (associated with canonical $\mathrm{Wg}$ signaling), it did not rescue the PCP function of Dsh, displaying classical PCP defects very similar to clones of $d s h^{\text {v26 }}$ in the eye (as shown here) and the wing (not shown). (D) Schematic summary of $d s h^{\mathrm{v} 26} / \mathrm{Y}$; $d s h-D s h G F P Y-\mathrm{xxx}-\mathrm{F}$ rescues: Note that, except for a DshY473F (and DshY473.474F), all other Tyr mutations fully rescued lethality and PCP defects, behaving like DshWT. Conserved Tyr residues are marked in red. $(E-F) \mathrm{Dsh}^{\mathrm{Y} 473 \mathrm{~F}}$ affects PCP-specific membrane association of Dsh, as detected in $30 \mathrm{~h}$ APF (after puparium formation) pupal wing cells. (E-E") $d s h^{\mathrm{v} 26} / Y$; $d s h-D s h G F P /+$ stained with anti-Fmi (red in $\left.E, E^{\prime}\right)$ and anti-GFP (DshGFP; green in $E$; grayscale in $E^{\prime \prime}$ ). Expression of $d s h$ DshGFP completely rescued the $d s h^{v 26} / Y$ mutant and displayed wild-type asymmetric membrane localization within the proximal-distal axis. $\left(F-F^{\prime \prime}\right) d s h^{v 26} / Y$; dsh-DshGFP $P^{Y 473 F} /+$ stained with anti-Fmi (red in $F, F^{\prime}$ ) and anti-GFP (DshGFP; green in $F$; grayscale in $\left.F^{\prime \prime}\right) .\left(F, F^{\prime \prime}\right)$ Note the markedly reduced Dsh membrane association, which is critical for Dsh function in PCP signaling. $\left(G, G^{\prime}\right)$ In vitro kinase assay with purified $\mathrm{Abl}$ and DEP-Y473F/C-term (shown in G). The top panel shows an autoradiograph of a kinase assay, where DEP-Y473F/C-term shows reduced phosphorylation as compared with wild-type DEP/C-term. The bottom panel shows Coomassie staining of the same gel to reveal equal loading. $\left(G^{\prime}\right)$ Quantification of the of DEP/C-term and DEP-Y473F/C-term phosphorylation (ratio of DEP/C-term and DEP-Y473F/C-term phosphorylated to the total amount of DEP/C-term and DEPY473F/C-term loaded) by purified Abl kinase. Note the significant reduction in Abl phosphorylation with DEP-Y473F/ $\mathrm{C}$-term as compared with wild-type DEP/C-term. Values shown represent the average $\pm \mathrm{SD}(n=3) \cdot\left({ }^{*}\right) P<0.01$, DEP-Y473F C-term compared with the wild-type DEP/C-term. 
$\mathrm{G}^{\prime}$ showing reduction in phosphorylation on DEP-Y473F/ C-term as compared with wild-type DEP/C-term). Taken together, these data establish that the Abl target site Tyr473 is physiologically required for Dsh function in PCP, and that the DshY473F mutant behaves like a PCPspecific allele of $d s h$.

\section{Mouse Abl family kinases affect Dvl phosphorylation and its localization}

To explore a conserved role for mammalian Abl kinases in regulating $\mathrm{Fz} / \mathrm{Dsh}-\mathrm{PCP}$ signaling, we used MEFs. There are three Dsh mouse homologs (Dvl1, Dvl2, and Dvl3) (Wang et al. 2006), while there are two Abl family members: mAbl1 and mAbl2 (also called Abl-related gene or Arg) (Kruh et al. 1990). Intriguingly, Dvl2 knockout mice share similar phenotypes with $\mathrm{Abl}^{-/-}, \mathrm{Abl2}^{-/-}$ double-mutant mice in that they both display neural tube closure defects during early stages of development (Koleske et al. 1998; Hamblet et al. 2002). Previous studies have suggested that phosphorylation of Dvl proteins can be resolved into two closely migrating bands corresponding to different phosphorylation states (Fig. 7A, lane 1; Gonzalez-Sancho et al. 2004; Corbit et al. 2008). Abl1 ${ }^{-1-}$, Abl2 ${ }^{-1-}$ double-mutant MEFs showed markedly reduced phosphorylation of Dvl2 and Dvl3, evident by the absence of the slower migrating band as compared with wild-type MEFs (Fig. 7A,B, lane 2). These data suggest that Dsh/Dvl protein phosphorylation by Abl kinases is conserved.

Activation of $\mathrm{Fz}$ receptors by Wnts induces phosphorylation of Dvl proteins (Willert et al. 1997; Sun et al. 2001; Liu et al. 2005; Wallingford and Habas 2005). As $A b 11^{-1-}$, $A b 12^{-/-}$double knockout MEFs showed reduced Dvl2/ Dvl3 phosphorylation, we tested whether this change in phosphorylation is associated with a change in canonical and/or PCP Wnt signaling. For canonical Wnt signaling, two assays were used: (1) the levels of uncomplexed $\beta$-catenin, and (2) TOP flash reporter activity. As reported, stimulation with Wnt3a but not Wnt5a resulted in increased uncomplexed $\beta$-catenin levels (Fig. 7C; Liu et al. 2005) and TOP flash activity in wild-type MEFs (Fig. 7D). These Wnt3A-induced effects were not compromised in the $A b 11^{-1-}, A b l 2^{-/-}$knockout MEFs (Fig. $7 \mathrm{C}, \mathrm{D})$. Taken together, these results suggest that canonical Wnt signaling remains largely unaffected in the absence of Abl kinase activity, consistent with our data from Drosophila, indicating that there is a specific requirement for $\mathrm{Abl}$ in Dsh phosphorylation in PCP signaling, but not in canonical Wnt signaling.

Membrane recruitment of Dsh is an important aspect of both Wnt signaling pathways: canonical and PCP (Axelrod 2001; Seifert and Mlodzik 2007). In particular, in Fz/PCP signaling, Dsh is stably recruited to the membrane and requires additional factors besides $\mathrm{Fz}$ for this localization (Wu et al. 2008; Simons et al. 2009). Thus, although there is no transcriptional reporter that can be used as a faithful readout for PCP signaling, stable membrane association of Dsh is a hallmark of PCP signaling and can be used as a signaling readout for Dsh
(Wallingford and Habas 2005). As we detected a reduction in Dvl2 and Dvl3 phosphorylation in $A b l 1^{-/-}$, $A b 12^{-/-}$knockout MEFs, we hypothesized that this might correlate with a change in subcellular localization of endogenous Dvl proteins. Anti-Dvl2 staining in wildtype MEFs revealed that a significant portion was associated with the plasma membrane (Fig. 7E-E"). Strikingly, in $A b 11^{-/-}, A b l 2^{-/-}$mutant MEFs, Dvl2 was localized predominantly to cytoplasmic puncta rather than to cell membranes (Fig. 7F-F"). These results suggest that Abl kinase phosphorylation of Dvl proteins is required for their stable membrane localization, providing molecular insight into how Abl may be regulating PCP signaling.

\section{Discussion}

We provide evidence for a specific role of tyrosine phosphorylation of Dsh by Abl family kinases in $\mathrm{Fz} /$ Dsh-PCP signaling. We demonstrate that $\mathrm{dAbl}$ is required for R3/R4 fate specification. $d A b l$ interacts with $f z$ and $d s h$ genetically in PCP signaling. Biochemical experiments indicate that $\mathrm{dAbl}$ binds Dsh and phosphorylates it on Tyr473 within the DEP domain, which has been specifically implicated in PCP signaling and is largely dispensable for canonical Wnt/Wg signaling (Boutros and Mlodzik 1999; Wallingford and Habas 2005). Our data further show that Abl kinases do not affect canonical Wnt signaling in either Drosophila or MEFs. Taken together, our data indicate that Abl family kinases positively regulate PCP signaling by affecting Dsh/Dvl family members via phosphorylation of Tyr473. Abl family kinases appear to provide a molecular gating mechanism to increase the capability of Dsh/Dvl proteins to signal via the Fz/Dsh-PCP pathway (Fig. 7G, see model). Our data suggest the possibility that this function of $\mathrm{Abl}$ family kinases might be conserved from flies to mammals, as similar effects were observed with mammalian Abl and Dvl family members.

\section{The role of Abl family kinases in PCP establishment}

Most Abl studies in mammalian cell culture have focused on their role in tumor formation. Little is known about Abl's normal physiological roles during development, except in the context of junctional stability and cytoskeletal events (see above). Previous studies in the Drosophila eye established that dAbl is expressed dynamically in all photoreceptors, and $d A b l$ mutant flies have a rough eye phenotype with significant photoreceptor loss (Henkemeyer et al. 1987). Its potential role in cell fate specification has not been addressed. We performed a detailed analysis of the $d A b l$ eye phenotype in the context of PCP establishment and R3/R4 specification, and demonstrate that $\mathrm{dAbl}$ is required for specification of both R3-R4 cells. Differential activation of Fz/PCP signaling specifies R3 and leads to the activation of Notch signaling in the neighboring R4 to induce its proper fate /Cooper and Bray 1999; Fanto and Mlodzik 1999). Our data suggest that $d A b 1$ is required in $\mathrm{R} 3$ for fate specification via its 
A

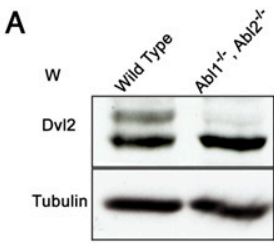

B

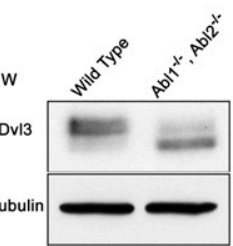

C

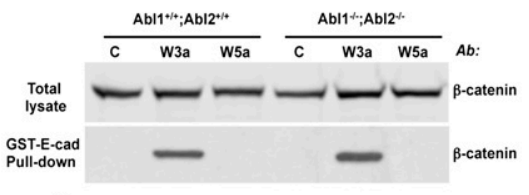

D
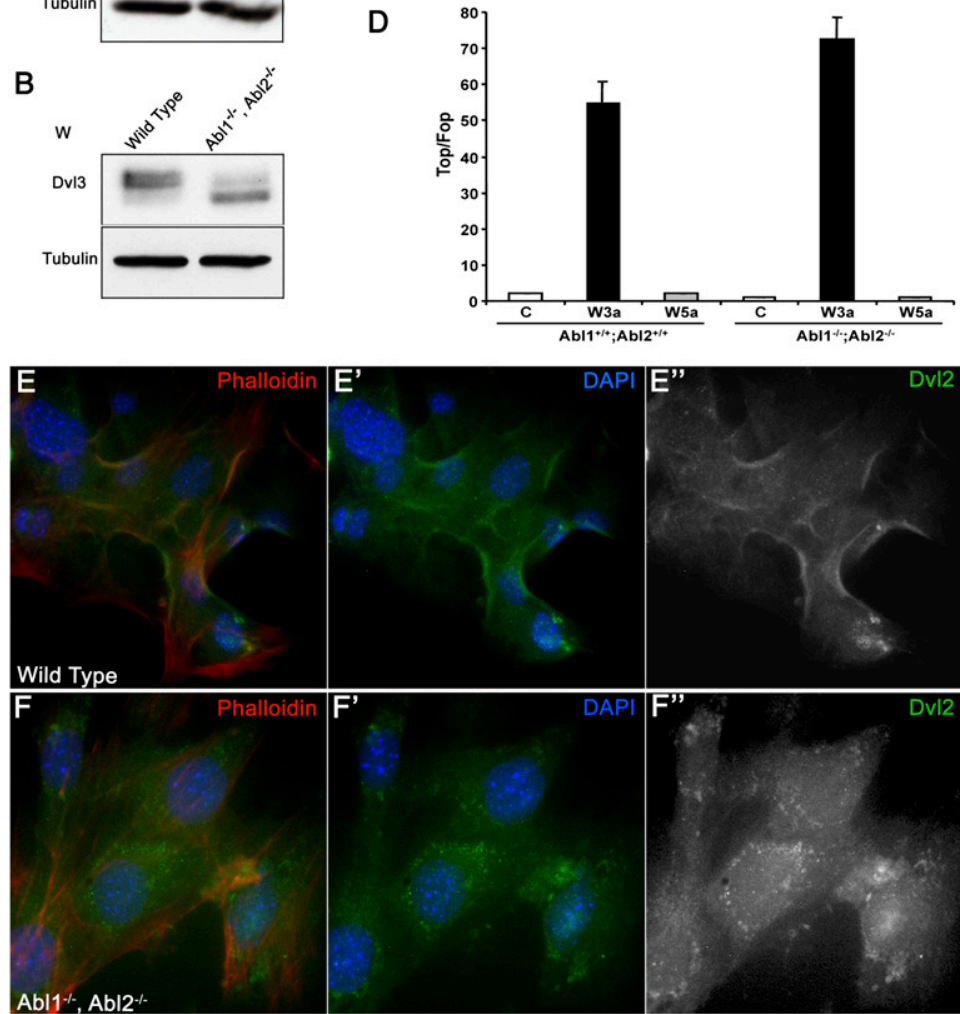

\section{G}

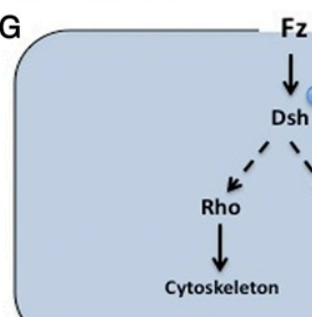

Cytoskeleton

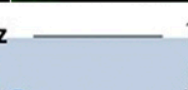

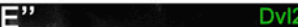

Figure 7. Mouse Abl kinases affect Dvl phosphorylation and its localization, without affecting canonical Wnt signaling. $(A, B)$ Western blot for mammalian Dvl2 $(A)$ and Dvl3 $(B)$ from wild-type and $A b 11^{-}, A b l 2^{-}$ knockout MEFs. The mutant MEF's show reduced phosphorylation of both Dvls tested (right lanes) as compared with wild-type MEFs (left lanes). $\gamma$-Tubulin serves as loading control. (C) Effects of Wnt3a and Wnt5a on canonical Wnt signaling readouts in wildtype and $\mathrm{Abl}^{-/-} ; \mathrm{Abl2}^{-/-}$knockout MEFs. Wild-type or $A b 11^{-1-} ; A b l 2^{-1-}$ MEFs were treated for $3 \mathrm{~h}$ with control, Wnt3a, or Wnt5a conditioned media, and the amount of uncomplexed $\beta$-catenin was measured by pull-down with GST-E-cadherin beads, followed by immunoblot with anti- $\beta$-catenin antibody. (Top panel) As control, the $\beta$-catenin levels in the total lysates are shown. (D) Effects of Wnt3a and Wnt5a on $\beta$-catenin/ TCF reporter in wild-type and $A b 1^{-/-} ; A b l 2^{-/-}$knockout MEFs. Cells were infected with either TOP-luciferase or FOP-luciferase lentiviruses and the renilla lentivirus, as an internal control for infection efficiency. The cells were replated and treated overnight with control, Wnt3a, or Wnt5a conditioned media, and the reporter activity was calculated by dividing the TOP/ renilla ratio by the FOP/renilla ratio. Values shown represent the average $\pm \mathrm{SD}(n=3)$ from a representative experiment. $\left(E-E^{\prime \prime}\right)$ Endogenous localization of Dvl2 (green in $\mathrm{E}_{1} \mathrm{E}^{\prime}$; grayscale in $E^{\prime \prime}$ ) in wild-type MEFs stained with phalloidin (red, highlighting cell membranes) and DAPI (blue, marking nuclei). Much of Dvl2 is localized to the plasma membrane. $\left(F-F^{\prime \prime}\right)$ Localization of endogenous Dvl2 (green in $F, F^{\prime}$; grayscale in $F^{\prime \prime}$ ) in $A b 1^{-/-}$; $A b l 2^{-/-}$MEFs stained as in $E-E^{\prime \prime}$. Note the loss of Dvl2 from membranes and the appearance of cytoplasmic puncta staining of Dvl2. (G) Schematic of proposed model where Abl phosphorylates Dsh, thereby "activating" it toward Fz/PCP signaling, while the canonical Wnt pathway remains largely unaffected. As such, Abl provides a gating mechanism to promote Dsh/PCP signaling.

interaction with Dsh and positive input into $\mathrm{Fz} / \mathrm{PCP}$ signaling. This Abl function appears to be common to $\mathrm{Fz} / \mathrm{PCP}$ signaling in general, as $\mathrm{Fz}$ and $\mathrm{dAbl}$ also synergize in PCP establishment in the wing. Moreover, dAbl phosphorylation of DshTyr473 is essential for Dsh PCP function in general. In vertebrates, Abl kinases affect Dvl localization in MEFs, and $A b 11^{-/-}, A b l 2^{-/-}$mice display similar phenotypes as $d v 11^{-/-}, d v 12^{-/-}$mice, with severe open neural tube defects in 9.5-d-post-coitum (dpc) embryos (Koleske et al. 1998; Hamblet et al. 2002). The requirement of $\mathrm{dAbl}$ in $\mathrm{R} 4$ specification remains obscure, as Fz/PCP signaling is not required in R4 (Mlodzik 2002). A likely explanation derives from studies of $\mathrm{dAbl}$ in noncanonical Notch signaling (Giniger 1998), where dAbl has been suggested to act downstream from Notch. Although the role of Notch in the developing eye has focused on canonical $\mathrm{Su}(\mathrm{H})$-dependent Notch activity (Tomlinson and Struhl 1999), it is quite likely that Abl could modulate Notch signaling activity in R4. Further work will be needed to explain the role of dAbl in R4 fate specification and associated Notch signaling.

\section{Dsh phosphorylation by $A b l$}

As described above, Dsh contains three highly conserved domains and a stretch of basic residues and several serine/ threonine-rich regions between the DIX and PDZ domains, as well as a proline-rich region downstream from the PDZ domain, which encodes a class I consensus sequence for an SH3-binding protein. Many proteins that have been shown to bind Dsh/Dvl bind to Dsh in the PDZ domain. Abl binding, however, maps to the proline-rich 
region of Dsh just C-terminal to the PDZ domain, while the PDZ domain alone showed no binding. Different Dsh domain requirements are known for canonical and PCP signaling (Boutros et al. 1998; Wallingford and Habas 2005). While the DIX domain functions exclusively in canonical Wnt signaling (Kishida et al. 1999), the DEP domain is required for PCP signaling and, in particular, stable membrane association (Wu et al. 2008; Simons et al. 2009). Our results indicate that dAbl phosphorylates Dsh at Tyr473 (and possibly other Tyr residues in the DEP/ C-term region). Phosphorylation of Dsh-Tyr473 is unique, as $\mathrm{dAbl}$ is a tyrosine kinase and all previously analyzed Dsh kinases have been serine/threonine kinases.

\section{Abl in Fz/Dsh-PCP signaling vs. canonical Wnt signaling}

Wnt signaling is important in diverse physiological processes and, when deregulated, often leads to disease states (Clevers 2006; Simons and Mlodzik 2008). Studies in model organisms have unraveled two conserved pathways, now referred to as canonical Wnt/Wg signaling and Wnt-Fz/PCP signaling (additional signaling pathways or branches are likely to exist) (Semenov et al. 2007). The canonical Wnt signal is transduced via Fz family receptors (along with the LRP5/6 coreceptors), leading to DshAxin complex formation, which in turn causes the stabilization of cytoplasmic $\beta$-catenin and allows gene transcription (Clevers 2006). InFz/PCP signaling, Dsh is recruited to the membrane in an Axin- and LRP5/ 6-independent manner and acts on different downstream effectors, depending on the cellular context (Klein and Mlodzik 2005). The mechanism of pathway-specific Dsh "activation" is poorly understood, and, similarly, the question as to how the individual pathways are specifically activated at the level of either $\mathrm{Fz}$ or Dsh remains unresolved. Our study highlights the importance of Abl in the context of Fz/PCP signaling at the level of Dsh/Dvl. At the level of both Abl mutants as well as the DshY473F phosphorylation mutant, canonical Wnt/Wg signaling remains unaffected, while PCP signaling is defective. As the signal that activates $\mathrm{dAbl}$ in this context is not known, it is possible that $\mathrm{Abl}$ acts in a permissive manner in PCP signaling. In conclusion, our study provides evidence for Dsh tyrosine phosphorylation and a role of $\mathrm{Abl}$ in PCP signaling; further studies will be needed to establish a full framework for the regulation of $\mathrm{Abl}$ in PCP signaling and in the biology of Dsh.

\section{Materials and methods}

\section{Drosophila stocks}

Flies were grown at the indicated temperatures. The following $d A b l$ stocks were used: $U A S-A b l$ and $D f(3 L) s t-j 7$ (E. Giniger), $U A S-d A b l-I R(X)$ and UAS-dAbl-IR(III) (Vienna Drosophila RNAi Center and Bloomington Stock Center), and $d A b 1^{1}$ and $d A b l^{2}$ (Bloomington Stock Center). Clonal and overexpression studies of dAbl were performed using standard FLP/FRT and GAL4/UAS techniques.

\section{Immunofluorescence and histology}

Third instar larvae were dissected in ice-cold PBS and fixed in $4 \%$ paraformaldehyde for $20 \mathrm{~min}$. After washing in PBT $10.1 \%$ Triton), dissected discs were stained with rabbit anti- $\beta$-Gal (Cappel), mouse anti-GFP (Molecular Probes), and rat anti-Elav (Developmental Studies Hybridoma Bank [DSHB]) antibodies followed by three washes in PBT, and were incubated with secondary antibody for $2 \mathrm{~h}$. Subsequently, discs were washed in PBT and mounted in Vectashield (Vector Laboratories).

For adult eye sections, the eyes were prepared, fixed, embedded, and sectioned as described (Gaengel and Mlodzik 2008). For each genotype, three to six eyes were sectioned and at least 400 individual ommatidia were scored.

MEFs were grown in DMEM supplemented with $10 \%$ fetal bovine serum and antibiotics. For immunofluorescence, MEFS were gown in chamber slides (Nunc) and cells were fixed in $4 \%$ paraformaldehyde. After washing in PBS, cells were incubated with anti-mouse Dvl2 and Dvl3 antibodies (Santa Cruz Biotechnology) overnight at $4^{\circ} \mathrm{C}$ followed by washes with PBS. Cells were then incubated with secondary antibodies and Hoechst to stain the nuclei for $2 \mathrm{~h}$ at room temperature, followed by washes in PBS. Cells were mounted in Vectashield (Vector Laboratories).

\section{Western blotting and coimmunoprecipitation}

S2 and 293T cells were grown in Schneider medium and DMEM (Gibco) using standard procedures. Coimmunoprecipitation experiments were carried out as described previously: In brief, cells were transfected with Dsh-Myc and/or Abl-HA or Abl-KD-HA using Cellfectin (Invitrogen) for S2 cells or Fugene transfection reagent for 293T cells (Roche), harvested after $48 \mathrm{~h}$, and lysed in ice-cold lysis buffer $(50 \mathrm{mM}$ Tris- $\mathrm{HCl}$ at $\mathrm{pH} 8.0,150 \mathrm{mM}$ $\mathrm{NaCl}, 1 \mathrm{mM}$ EDTA, $5 \mathrm{mM} \beta$-glycerophosphate, protease inhibitor cocktail [Roche], 1\% Triton X-100). Lysed samples were immunoprecipitated using mouse anti-Myc (Santa Cruz Biotechnology). Western blots were carried out with immunoprecipitated samples using rabbit anti-HA (Sigma), mouse anti-myc (Santa Cruz Biotechnology), or phosphotyrosine (Millipore) antibodies.

\section{GST pull-down and kinase assays}

Purified GST-Dsh truncations, described previously (Jenny et al. 2005), were used for pull-down and in vitro kinase assays. For GST pull-down assays, $1 \mu \mathrm{g}$ of GST-Dsh proteins along with GST beads were incubated with $5 \mu \mathrm{L}$ of $S^{35}$-labeled in vitro translated dAbl (according to the manufacturer's instructions; Promega) in Buffer PD $(50 \mathrm{mM}$ Tris- $\mathrm{HCl}$ at $\mathrm{pH}$ 7.6, $150 \mathrm{mM} \mathrm{KCl}, 0.5 \%$ Triton $\mathrm{X}-100,1 \mathrm{mM}$ DTT, protease inhibitor cocktail) for $60 \mathrm{~min}$ at $4^{\circ} \mathrm{C}$. Beads were washed three times in ice-cold buffer PD before addition of sample buffer.

In vitro kinase assays were preformed using $1 \mu \mathrm{g}$ of GST-Dsh truncations incubated in $50 \mathrm{U}$ of purified Abl kinase (New England Biolabs) for $30 \mathrm{~min}$ (according to the manufacturer's instructions). Proteins were resolved on SDS PAGE followed by autoradiography. GST-Crk (Cell Signaling Technology) was used as a positive control.

\section{Free $\beta$-catenin and lentiviral TCF reporter assays}

Wild-type and $\mathrm{AbI}^{-1-} ; A b 12^{-1-}$ MEFs were treated for $3 \mathrm{~h}$ with control, Wnt3a, or Wnt5a conditioned media collected from L cells (American Type Culture Collection [ATCC]; according to ATCC protocols), and the amounts of uncomplexed $\beta$-catenin were measured by the GST-E-cadherin-binding assay. Briefly, 
bacterially expressed GST-E-cadherin was bound to glutathioneSepharose beads (Amersham) and incubated with equal amount of cell lysate. After pull-down, the samples were subjected to Western analysis using monoclonal anti- $\beta$-catenin antibody (BD Biosciences).

For lentiviral TCF reporter experiments, vectors containing 14 wild-type (MegaTop-LentiLuc) or mutant (MegaFop-LentiLuc) TCF consensus elements driving firefly luciferase were used in combination with a lentivirus containing renilla luciferase under the PGK promoter. For lentivirus production, 293T cells were cotransfected with the lentiviral vector and pCMV $\Delta 8.91$ and pMD VSV-G plasmids (kindly provided by I. Weissman) using Fugene 6 (Roche) according to the manufacturer's instructions. Two days after transfection, the conditioned media were collected, supplemented with $8 \mu \mathrm{g} / \mathrm{mL}$ polybrene, and added for overnight incubation to freshly plated cells for infection. Wildtype and $\mathrm{Abl}^{-1-} ; \mathrm{Abl2} \mathrm{2}^{-/-} \mathrm{MEFs}$ were infected with MegaTopLentiLuc or MegaFop-LentiLuc, together with $1 / 20$ vol of renilla luciferase virus for normalization. MEFs with stable integration of the reporters were treated overnight with control, Wnt3a, or Wnt5a conditioned media, and luciferase activity was measured using the Dual-Luciferase Reporter Assay System (Promega). Reporter activity was calculated by dividing the MegaTOP/ renilla ratio by the $\mathrm{MegaFOP} /$ renilla ratio.

\section{Acknowledgments}

We thank the Bloomington Stock Center, DSHB, Edward Giniger, David Van Vactor, Mark Peifer, and Mark Seeger for various fly stocks, plasmid DNA, and antibodies; and all members of the Mlodzik laboratory for helpful suggestions and discussion. We are grateful to Andreas Jenny for providing GST-Dsh constructs, and Anthony Koleske for Abl knockout MEFs. We also thank William Gault for critical reading of the manuscript, and Joyce Lau, Sophy Okello, and Susanna Franks for technical help. J.S. thanks Nadinath Nillegoda, Dipankar Dutta, and Maneesha Chhikara for their helpful comments and suggestions. Confocal laser microscopy was performed at the MSSM Microscopy SRF, supported by a NIH/NCI shared instrumentation grant. This research was supported by NIH grants R01 EY013256 (to M.M.) and R01 CA091672 (to S.A.A.).

\section{References}

Axelrod JD. 2001. Unipolar membrane association of Dishevelled mediates Frizzled planar cell polarity signaling. Genes Dev 15: 1182-1187.

Bennett RL, Hoffmann FM. 1992. Increased levels of the Drosophila Abelson tyrosine kinase in nerves and muscles: Subcellular localization and mutant phenotypes imply a role in cell-cell interactions. Development 116: 953-966.

Boutros M, Mlodzik M. 1999. Dishevelled: At the crossroads of divergent intracellular signaling pathways. Mech Dev 83: $27-37$.

Boutros M, Paricio N, Strutt DI, Mlodzik M. 1998. Dishevelled activates JNK and discriminates between JNK pathways in planar polarity and wingless signaling. Cell 94: 109-118.

Clevers H. 2006. Wnt $/ \beta$-catenin signaling in development and disease. Cell 127: 469-480.

Cong F, Schweizer L, Varmus H. 2004. Casein kinase I $\varepsilon$ modulates the signaling specificities of dishevelled. Mol Cell Biol 24: 2000-2011.

Cooper MT, Bray SJ. 1999. Frizzled regulation of Notch signalling polarizes cell fate in the Drosophila eye. Nature 397: 526-530.
Corbit KC, Shyer AE, Dowdle WE, Gaulden J, Singla V, Chen $\mathrm{MH}$, Chuang PT, Reiter JF. 2008. Kif3a constrains $\beta$-catenindependent Wnt signalling through dual ciliary and nonciliary mechanisms. Nat Cell Biol 10: 70-76.

Fanto M, Mlodzik M. 1999. Asymmetric Notch activation specifies photoreceptors R3 and R4 and planar polarity in the Drosophila eye. Nature 397: 523-526.

Fanto M, Weber U, Strutt DI, Mlodzik M. 2000. Nuclear signaling by Rac and Rho GTPases is required in the establishment of epithelial planar polarity in the Drosophila eye. Curr Biol 10: 979-988.

Fox DT, Peifer M. 2007. Abelson kinase (Abl) and RhoGEF2 regulate actin organization during cell constriction in Drosophila. Development 134: 567-578.

Gaengel K, Mlodzik M. 2008. Microscopic analysis of the adult Drosophila retina using semithin plastic sections. Methods Mol Biol 420: 277-287.

Giniger E. 1998. A role for Abl in Notch signaling. Neuron 20: $667-681$.

Gonzalez-Sancho JM, Brennan KR, Castelo-Soccio LA, Brown AM. 2004. Wnt proteins induce dishevelled phosphorylation via an LRP5/6- independent mechanism, irrespective of their ability to stabilize $\beta$-catenin. Mol Cell Biol 24: 4757-4768.

Grevengoed EE, Loureiro JI, Jesse TL, Peifer M. 2001. Abelson kinase regulates epithelial morphogenesis in Drosophila. J Cell Biol 155: 1185-1198.

Hamblet NS, Lijam N, Ruiz-Lozano P, Wang J, Yang Y, Luo Z, Mei L, Chien KR, Sussman DJ, Wynshaw-Boris A. 2002. Dishevelled 2 is essential for cardiac outflow tract development, somite segmentation and neural tube closure. Development 129: 5827-5838.

Henkemeyer MJ, Gertler FB, Goodman W, Hoffmann FM. 1987. The Drosophila Abelson proto-oncogene homolog: Identification of mutant alleles that have pleiotropic effects late in development. Cell 51: 821-828.

Jenny A, Reynolds-Kenneally J, Das G, Burnett M, Mlodzik M. 2005. Diego and Prickle regulate Frizzled planar cell polarity signalling by competing for Dishevelled binding. Nat Cell Biol 7: 691-697.

Kishida S, Yamamoto H, Hino S, Ikeda S, Kishida M, Kikuchi A. 1999. DIX domains of Dvl and axin are necessary for protein interactions and their ability to regulate $\beta$-catenin stability. Mol Cell Biol 19: 4414-4422.

Klein TJ, Mlodzik M. 2005. Planar cell polarization: An emerging model points in the right direction. Annu Rev Cell Dev Biol 21: 155-176.

Klein TJ, Jenny A, Djiane A, Mlodzik M. 2006. CKI $\epsilon$ /discs overgrown promotes both $\mathrm{Wnt}-\mathrm{Fz} / \beta$-catenin and $\mathrm{Fz} / \mathrm{PCP}$ signaling in Drosophila. Curr Biol 16: 1337-1343.

Koleske AJ, Gifford AM, Scott ML, Nee M, Bronson RT, Miczek KA, Baltimore D. 1998. Essential roles for the Abl and Arg tyrosine kinases in neurulation. Neuron 21: 1259-1272.

Kruh GD, Perego R, Miki T, Aaronson SA. 1990. The complete coding sequence of arg defines the Abelson subfamily of cytoplasmic tyrosine kinases. Proc Natl Acad Sci 87: 5802-5806.

Lawrence PA, Struhl G, Casal J. 2007. Planar cell polarity: One or two pathways? Nat Rev Genet 8: 555-563.

Liu G, Bafico A, Aaronson SA. 2005. The mechanism of endogenous receptor activation functionally distinguishes prototype canonical and noncanonical Wnts. Mol Cell Biol 25: 3475-3482.

MacDonald BT, Tamai K, He X. 2009. Wnt/ $\beta$-catenin signaling: Components, mechanisms, and diseases. Dev Cell 17: 9-26.

Mlodzik M. 2002. Planar cell polarization: Do the same mechanisms regulate Drosophila tissue polarity and vertebrate gastrulation? Trends Genet 18: 564-571. 
Singh et al.

Munoz-Descalzo S, Gomez-Cabrero A, Mlodzik M, Paricio N. 2007. Analysis of the role of the Rac/Cdc42 GTPases during planar cell polarity generation in Drosophila. Int I Dev Biol 51: 379-387.

Seifert JR, Mlodzik M. 2007. Frizzled/PCP signalling: A conserved mechanism regulating cell polarity and directed motility. Nat Rev Genet 8: 126-138.

Semenov MV, Habas R, Macdonald BT, He X. 2007. SnapShot: Noncanonical Wnt signaling pathways. Cell 131: 1378.

Simons M, Mlodzik M. 2008. Planar cell polarity signaling: From fly development to human disease. Annu Rev Genet 42: 517-540.

Simons M, Gault WJ, Gotthardt D, Rohatgi R, Klein TJ, Shao Y, Lee HI, Wu AL, Fang Y, Satlin LM, et al. 2009. Electrochemical cues regulate assembly of the Frizzled/Dishevelled complex at the plasma membrane during planar epithelial polarization. Nat Cell Biol 11: 286-294.

Strutt H, Price MA, Strutt D. 2006. Planar polarity is positively regulated by casein kinase I $\epsilon$ in Drosophila. Curr Biol 16: 1329-1336.

Sun TQ, Lu B, Feng JJ, Reinhard C, Jan YN, Fantl WJ, Williams LT. 2001. PAR-1 is a Dishevelled-associated kinase and a positive regulator of Wnt signalling. Nat Cell Biol 3: 628-636.

Tomlinson A, Struhl G. 1999. Decoding vectorial information from a gradient: Sequential roles of the receptors Frizzled and Notch in establishing planar polarity in the Drosophila eye. Development 126: 5725-5738.

Wallingford JB, Habas R. 2005. The developmental biology of Dishevelled: An enigmatic protein governing cell fate and cell polarity. Development 132: 4421-4436.

Wang Y, Nathans J. 2007. Tissue/planar cell polarity in vertebrates: New insights and new questions. Development 134: 647-658.

Wang J, Hamblet NS, Mark S, Dickinson ME, Brinkman BC, Segil N, Fraser SE, Chen P, Wallingford JB, Wynshaw-Boris A. 2006. Dishevelled genes mediate a conserved mammalian PCP pathway to regulate convergent extension during neurulation. Development 133: 1767-1778.

Willert K, Brink M, Wodarz A, Varmus H, Nusse R. 1997. Casein kinase 2 associates with and phosphorylates dishevelled. EMBO J 16: 3089-3096.

Wu J, Mlodzik M. 2009. A quest for the mechanism regulating global planar cell polarity of tissues. Trends Cell Biol 19: 295-305.

Wu J, Jenny A, Mirkovic I, Mlodzik M. 2008. Frizzled-Dishevelled signaling specificity outcome can be modulated by Diego in Drosophila. Mech Dev 125: 30-42.

Xiong W, Dabbouseh NM, Rebay I. 2009. Interactions with the abelson tyrosine kinase reveal compartmentalization of eyes absent function between nucleus and cytoplasm. Dev Cell 16: $271-279$. 


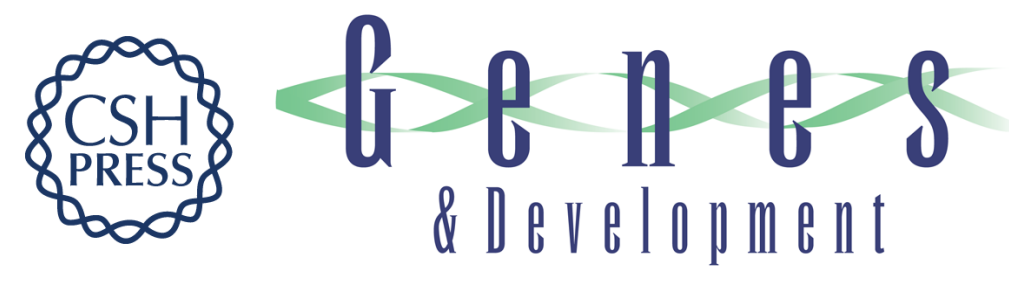

\title{
Abelson family kinases regulate Frizzled planar cell polarity signaling via Dsh phosphorylation
}

\author{
Jaskirat Singh, Wang A. Yanfeng, Luca Grumolato, et al.
}

Genes Dev. 2010, 24: originally published online September 13, 2010

Access the most recent version at doi:10.1101/gad.1961010

\section{Supplemental http://genesdev.cshlp.org/content/suppl/2010/09/03/gad.1961010.DC1 Material \\ Related Content \\ References \\ This article cites 42 articles, 14 of which can be accessed free at: http://genesdev.cshlp.org/content/24/19/2157.full.html\#ref-list-1 \\ Articles cited in: \\ http://genesdev.cshlp.org/content/24/19/2157.full.html\#related-urls \\ License \\ Email Alerting \\ Service \\ Abl to Regulate PCP \\ Annalisa M. VanHook \\ Sci. Signal. October , 2010 3: ec313 \\ Receive free email alerts when new articles cite this article - sign up in the box at the top right corner of the article or click here.}

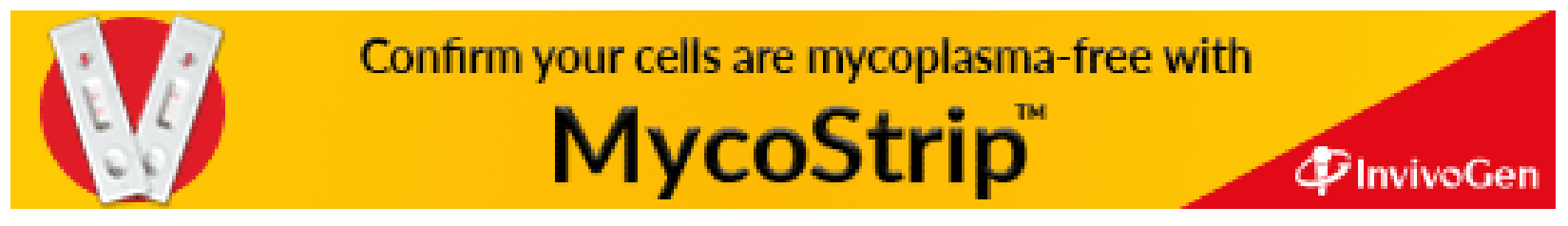

